\title{
Effects of Low Temperature and Low Light on Physiology of Tomato Seedlings
}

\author{
Yubo Yang1, Lingdi Dong1*, Linqi Shi'1, Jinghua Guo1, Yonggang Jiao1, Haizheng Xiong², \\ Ryan William Dickson², Ainong Shi²
}

${ }^{1}$ Institute of Economic Crops, Hebei Academy of Agricultural and Forestry Sciences, Shijiazhuang, China
${ }^{2}$ Department of Horticulture, University of Arkansas, Fayetteville, USA

Email: *donglingdi@163.com

How to cite this paper: Yang, Y.B., Dong, L.D., Shi, L.Q., Guo, J.H., Jiao, Y.G., Xiong, H.Z., Dickson, R.W. and Shi, A.N. (2020) Effects of Low Temperature and Low Light on Physiology of Tomato Seedlings. American Journal of Plant Sciences, 11, 162-179. https://doi.org/10.4236/ajps.2020.112013

Received: December 22, 2019

Accepted: February 17, 2020

Published: February 20, 2020

Copyright $\odot 2020$ by author(s) and Scientific Research Publishing Inc. This work is licensed under the Creative Commons Attribution International License (CC BY 4.0).

http://creativecommons.org/licenses/by/4.0/

\begin{abstract}
The physiological changes and the mechanism of stress tolerance in tomato were studied under low temperature and low light conditions. Two growth chamber experiments evaluated three temperatures regimes under standard and relatively low illumination levels with three tomato genotypes. Both experiments used a completely randomized split-plot design (CRD), with temperature regime as the main plot and tomato genotype as the split-plot. The three tomato varieties were "Fenyan No.1", "SV0313TG", and "Ousa". In both experiments, activity of superoxide dismutase (SOD) and peroxidases (POD) in tomato seedlings decreased under low temperature regime and the combination of low temperature and low light. Decreasing temperature had the greatest effect on the increase in enzyme activity. Decrease in POD activity was the greatest under low light and low temperature. The concentration of malondialdehyde (MDA) in plant tissue also decreased under low temperature $\left(20^{\circ} \mathrm{C} / 10^{\circ} \mathrm{C}\right.$ day/night $)$ compared to the standard temperature control $\left(25^{\circ} \mathrm{C} / 16^{\circ} \mathrm{C}\right.$ day/night $)$, but increased at $15^{\circ} \mathrm{C} / 5^{\circ} \mathrm{C}$ day/night temperatures in both experiments and was the greatest under the lowest light and temperature conditions. In both experiments, proline concentrations were the greatest under the standard light intensity (30,000 lux), and proline concentrations increased as temperature decreased. The content of soluble sugar decreased under only low temperature stress but increased under double stresses. The relative value of osmotic potential increased a little under low temperature stress but decreased under double stresses.
\end{abstract}

\section{Keywords}

Enzyme Activity, Malondialdehyde, Osmotic Potential, Peroxidases, Proline, Physiological Condition, Soluble Sugar, Stress Tolerance, Superoxide Dismutase 


\section{Introduction}

Tomato is the fourth largest vegetable crop in Hebei Province, China, and is often cultivated in the field and in protected unheated greenhouse structures for extended production [1]. In recent years, air pollution has become a major problem in Shijiazhuang City, Hebei Province, China, particularly in winter, where both natural light intensity and air temperature decrease as a result of smog conditions [2]. Dong et al. [3] observed in Shijiazhuang City that a continuous smog lasted 40 days during winter in 2015 , causing greenhouse temperature and light intensity to routinely decrease to below $10^{\circ} \mathrm{C}$ and 400 lux, respectively, during the day.

These temperatures and light intensities are well below recommended values for optimal tomato production and therefore result in decreased plant growth, fruit quality, and total yield [4] [5] [6] [7].

Past researchers have evaluated light intensity and temperature effects across a range of agronomic crop species [8] [9], and found that plant growth and biochemical analyses could be used to indicate plant stress tolerance to low light intensity and low temperature conditions. For example, reductions in root/shoot growth, photosynthetic rate, and plant biochemical markers such as malondialdehyde typical responses to low light and low temperature for crops such as melon, cucumbers, and tomato [10] [11] [12]. It may, therefore, be possible to measure relative differences in various plant growth and biochemical parameters as a technique for screening plant genotypes for tolerance to low light intensity and low temperature stress [13] [14].

Tomato cultivars used for production in Hebei Province, China, are adapted to high light intensity and a warm semi-tropical climate and may be particularly sensitive to lower light intensities and temperatures caused by air pollution and smog. In contrast, cultivars that were developed in northern climates with naturally lower light levels and temperatures, such as Northern Europe, may be more adapted to lower light and temperature conditions and may show greater tolerance to the adverse effects of air pollution in Shijiazhuang City.

The objective of this research was to 1) evaluate the effects of light intensity and air temperature on several physiological responses with three tomato cultivars and 2) determine if evaluating physiological responses could be used to screen tomatoes for tolerance to low light intensity and low temperature conditions. Two growth chamber experiments using a split-plot design evaluated three temperature regimes at two light intensities for effects on nutrition contents and osmotic potential. Two tomato cultivars were selected as common cultivars used in production, Hebei Province, China, and the third cultivar was selected from The Netherlands and was reported to have greater tolerance to low intensity and low temperature.

\section{Materials and Methods}

\subsection{Plant Material}

Tomato cultivars "SV0313TG" and "Ousa" were introduced from the United 
States with reports of low tolerance to low temperature and low light intensity whereas "Fenyan No.1" was originally imported from Netherlands and was reported to have moderate tolerance to low temperature and low light [3]. The three cultivars were further selected from the Economic Crops Research Institute of Hebei Academy of Agricultural and Forestry Sciences. The seed for each genotype was germinated in 72-cell plastic trays at one seed per cell.

\subsection{Experiment Design and Sampling}

Two experiments were conducted in 2015 in controlled-environment greenhouses at the High-tech Vegetable Science and Technology Park of Hebei Province, and the Physiology and Biochemistry Laboratory of the Economic Crops Research Institute of Hebei Academy of Agricultural and Forestry Sciences, China.

The first experiment was conducted under a standard light intensity of 30,000 Lux during an eight-hour photoperiod. The second experiment consisted of growing plants under a lower light intensity of 3000 Lux during the same eight-hour photoperiod regime (Table 1). Both experiments had three day/night temperature regimes that consisted of $25^{\circ} \mathrm{C} / 16^{\circ} \mathrm{C}, 20^{\circ} \mathrm{C} / 10^{\circ} \mathrm{C}$, and $15^{\circ} \mathrm{C} / 5^{\circ} \mathrm{C}$ (Table 1). Both experiments were arranged using a split-plot completely randomized design (CRD), with temperature treatment and tomato genotype as the main plot and split-plot factors, respectively. Once plants reached to the two-leaf stage, they were placed in different constant temperature incubators with different parameters set under treatments of low temperature and weak light tests. After continuous culture in the constant temperature incubators for 21 days, the upper part leaves of the tomato seedlings were taken immediately, and the physiological and biochemical indexes were determined in the laboratory according to different measurement methods.

\subsection{Phenotyping of Physiological and Biochemical Indexes}

After different temperature and light treatments, fully expanded and mature leaves from the middle and upper portion of tomato seedlings were sampled and

Table 1. Parameter setting of constant temperature incubator for different treatments under two experiments with normal and weak illuminations.

\begin{tabular}{ccccccc}
\hline Treatment & Period & Duration & $\begin{array}{c}\text { Relative } \\
\text { Humidity } \\
(\%)\end{array}$ & $\begin{array}{c}\text { Temperature } \\
\left({ }^{\circ} \mathrm{C}\right)\end{array}$ & $\begin{array}{c}\text { Exp. } 1 \\
\text { Normal } \\
\text { Illumination } \\
(\text { Lux })\end{array}$ & $\begin{array}{c}\text { Exp. } 2 \\
\text { Weak } \\
\text { Illumination } \\
\text { (Lux) }\end{array}$ \\
\hline \multirow{2}{*}{ CK } & Day & 8 & 75 & 25 & 30,000 & 3000 \\
& Night & 16 & 75 & 16 & 0 & 0 \\
& Day & 8 & 75 & 20 & 30,000 & 3000 \\
T1 & Night & 16 & 75 & 10 & 0 & 0 \\
& Day & 8 & 75 & 15 & 30,000 & 3000 \\
& Night & 16 & 75 & 5 & 0 & 0 \\
\hline
\end{tabular}


measurements of superoxide dismutase (SOD), peroxidases (POD), malondialdehyde (MDA), proline, soluble sugar, and osmotic potential were determined in leaf tissues. The SOD was determined using the nitroblue tetrazolium method as described by $\mathrm{Wu}$ [15]. Peroxidase content was determined using the concentration of guaiacol [16]. Malondialdehyde was determined by the concentration of thiobarbituric acid [16] and proline content was measured using the ninhydrin colorimetric method described by Zhang [17]. Soluble sugar concentration was determined using an anthrone colorimetric procedure [18], and osmotic potential in leaf tissue using the plasmolysis method [14].

Measurements of SOD, POD, MDA, soluble sugars and osmotic potential were replicated four times and analyzed using methods described by previous researchers (Dong et al., 2019; Fernandez, 1992; González, 1996; Saad et al., 2014) (Table 2 and Table 3): 1) the raw data were measured for each content based on its method; 2) absolute reduced value (CK-raw datum) for each tomato genotypes for each experiment, respectively; 3 ) relative reduction value (\%) $\left[100^{*}\right.$ (CK-raw datun)/CK]; and 4) relative value compared to $\mathrm{CK}$ as $100 \%$ $\left[100^{*}\right.$ (raw datun/CK)].

\subsection{Data Analysis}

Analysis of variance (ANOVA) with PROC GLM (SAS 9.4, SAS Institute, Cary, $\mathrm{NC}$ ) was used to evaluate genotype and temperature effects on tomato leaf SOD, POD, MDA, soluble sugars and osmotic potential for each light intensity. A student T-test was used for posthoc comparison of tomato genotypes based on multiple comparisons for the least square mean (LSM) at $\mathrm{P}=0.05$. Statistic charts were drowned only for the relative value of each index in two experiments using Microsoft Excel 2016.

\section{Results}

\subsection{Temperature Effects on the Activity and Content of Six Chemical Substances in Tomato under Normal Light}

In the Expt. 1 under standard light conditions, the low temperature had an effect on SOD, POD, MDA, soluble sugars, protein content, and leaf osmotic potential (Table 2, Figures 1-4).

Values for SOD and POD activity decreased in all the three tomato cultivars as the day/night temperature decreased. The value of the SOD and POD indicated the lower temperature was, the more enzyme activities decreased (Table 2). Averaged across cultivars, SOD was decreased by $24.8 \%$ and $35.8 \%$, and POD by $9.4 \%$ and $35.4 \%$ under $\mathrm{T} 1$ and $\mathrm{T} 2$, respectively (Table 2). When the CK was standardized to $100 \%$, the average SOD value was $75.2 \%$ and $64.2 \%$, and the POD was $90.6 \%$ and $64.6 \%$ under T1 and T2 conditions, respectively (Table 2, Figure 1). Trends were similar between cultivars (Table 2, Figures 2-4). For example, when CK was standardized to $100 \%$, the SOD value in SV0313TG was $79.7 \%$ and $69.6 \%$, and the POD was $89.1 \%$ and $69.1 \%$ under T1 and T2 conditions, 
Table 2. Temperature effects on the activities and contents of six chemical substances in three tomato cultivars under normal light condition from experiment one.

\begin{tabular}{|c|c|c|c|c|c|c|c|c|c|c|}
\hline Cultivar & Treatment & $\begin{array}{c}\text { SOD } \\
\text { (U/mg.protein) }\end{array}$ & $\begin{array}{l}\text { Relative } \\
\text { SOD } \\
\text { reduction } \\
\text { value (\%) }\end{array}$ & $\begin{array}{c}\text { Relative } \\
\text { SOD } \\
\text { value } \\
(\mathrm{CK} \text { as } \\
100)\end{array}$ & $\begin{array}{c}\text { POD } \\
(\mathrm{U} / \mathrm{mg} \cdot \text { protein } \cdot \mathrm{min})\end{array}$ & $\begin{array}{l}\text { Relative } \\
\text { POD } \\
\text { reduction } \\
\text { value (\%) }\end{array}$ & $\begin{array}{c}\text { Relative } \\
\text { POD value } \\
\text { (CK as 100) }\end{array}$ & $\begin{array}{c}\text { MDA } \\
(\mathrm{nmol} / \mathrm{mg} \cdot \mathrm{FW})\end{array}$ & $\begin{array}{l}\text { Relative } \\
\text { MDA } \\
\text { reduction } \\
\text { value (\%) }\end{array}$ & $\begin{array}{c}\text { Relative } \\
\text { MDA } \\
\text { value } \\
(\mathrm{CK} \text { as } \\
100)\end{array}$ \\
\hline \multirow{3}{*}{ SV0313TG } & $\mathrm{CK}$ & $13.8 \mathrm{a}$ & -- & 100 & $37.6 \mathrm{a}$ & -- & 100 & $11.2 \mathrm{~b}$ & -- & 100 \\
\hline & $\mathrm{T} 1$ & $11.0 \mathrm{a}$ & 20.3 & 79.7 & $33.5 \mathrm{a}$ & 10.9 & 89.1 & $10.7 \mathrm{~b}$ & 4.5 & 95.5 \\
\hline & $\mathrm{T} 2$ & $9.6 \mathrm{a}$ & 30.4 & 69.6 & $26.0 \mathrm{a}$ & 30.9 & 69.1 & $16.4 \mathrm{a}$ & -46.4 & 146.4 \\
\hline \multirow{3}{*}{ Ousa } & $\mathrm{CK}$ & $16.1 \mathrm{a}$ & -- & 100.0 & $42.3 \mathrm{a}$ & -- & 100.0 & $8.9 \mathrm{a}$ & -- & 100.0 \\
\hline & $\mathrm{T} 1$ & $11.5 \mathrm{~b}$ & 28.6 & 71.4 & $34.9 \mathrm{ab}$ & 17.5 & 82.5 & $8.8 \mathrm{a}$ & 1.1 & 98.9 \\
\hline & $\mathrm{T} 2$ & $10.1 \mathrm{~b}$ & 37.3 & 62.7 & $25.7 \mathrm{~b}$ & 39.2 & 60.8 & $9.8 \mathrm{a}$ & -10.1 & 110.1 \\
\hline \multirow{3}{*}{$\begin{array}{c}\text { Fenyan } \\
\text { No.1 }\end{array}$} & $\mathrm{CK}$ & $14.5 \mathrm{a}$ & -- & 100.0 & $49.4 \mathrm{a}$ & -- & 100.0 & $12.5 \mathrm{a}$ & -- & 100.0 \\
\hline & $\mathrm{T} 1$ & $10.9 \mathrm{ab}$ & 24.8 & 75.2 & $48.8 \mathrm{a}$ & 1.2 & 98.8 & $10.2 b$ & 18.4 & 81.6 \\
\hline & $\mathrm{T} 2$ & $8.8 \mathrm{~b}$ & 39.3 & 60.7 & $31.8 \mathrm{~b}$ & 35.6 & 64.4 & $10.4 \mathrm{~b}$ & 16.8 & 83.2 \\
\hline \multirow{3}{*}{ Average } & $\mathrm{CK}$ & 14.8 & -- & 100.0 & 43.1 & -- & 100.0 & 10.9 & -- & 100.0 \\
\hline & $\mathrm{T} 1$ & 11.1 & 24.8 & 75.2 & 39.1 & 9.4 & 90.6 & 9.9 & 8.9 & 91.1 \\
\hline & $\mathrm{T} 2$ & 9.5 & 35.8 & 64.2 & 27.8 & 35.4 & 64.6 & 12.2 & -12.3 & 112.3 \\
\hline \multirow[t]{2}{*}{ Cultivar } & Treatment & $\begin{array}{c}\text { Proline } \\
\mu \mathrm{g} /(\mathrm{g} \cdot \mathrm{FW})\end{array}$ & $\begin{array}{l}\text { Relative } \\
\text { Proline } \\
\text { reduction } \\
\text { value (\%) }\end{array}$ & $\begin{array}{c}\text { Relative } \\
\text { Proline } \\
\text { value } \\
(\mathrm{CK} \text { as } \\
100)\end{array}$ & $\begin{array}{c}\text { Soluble sugar } \\
\mathrm{mg} /(\mathrm{g} \cdot \mathrm{FW})\end{array}$ & $\begin{array}{c}\text { Relative } \\
\text { Soluble } \\
\text { sugar } \\
\text { reduction } \\
\text { value (\%) }\end{array}$ & $\begin{array}{c}\text { Relative } \\
\text { Soluble } \\
\text { sugar value } \\
\text { (CK as } 100)\end{array}$ & $\begin{array}{c}\text { Osmotic } \\
\text { potential } \\
(\mathrm{Mpa})\end{array}$ & $\begin{array}{l}\text { Relative } \\
\text { Osmotic } \\
\text { potential } \\
\text { reduction } \\
\text { value (\%) }\end{array}$ & $\begin{array}{c}\text { Relative } \\
\text { Osmotic } \\
\text { potential } \\
\text { value } \\
(\mathrm{CK} \text { as } \\
100)\end{array}$ \\
\hline & CK & $87.3 \mathrm{~b}$ & -- & 100.0 & $3.5 \mathrm{a}$ & -- & 100.0 & $-0.93 a$ & -- & 100.0 \\
\hline \multirow[t]{3}{*}{ SV0313TG } & $\mathrm{T} 1$ & $666.8 \mathrm{a}$ & -663.8 & 763.8 & $1.3 \mathrm{~b}$ & 62.9 & 37.1 & $-0.97 b$ & -4.3 & 104.3 \\
\hline & $\mathrm{T} 2$ & $864.0 \mathrm{a}$ & -889.7 & 989.7 & $1.4 \mathrm{~b}$ & 60.0 & 40.0 & $-0.97 b$ & -4.3 & 104.3 \\
\hline & $\mathrm{CK}$ & $101.8 \mathrm{~b}$ & -- & 100.0 & $3.5 \mathrm{a}$ & -- & 100.0 & $-0.88 \mathrm{a}$ & -- & 100.0 \\
\hline \multirow[t]{3}{*}{ Ousa } & $\mathrm{T} 1$ & $647.6 \mathrm{a}$ & -536.1 & 636.1 & $1.3 \mathrm{~b}$ & 62.9 & 37.1 & $-1.01 b$ & -14.8 & 114.8 \\
\hline & $\mathrm{T} 2$ & $811.2 \mathrm{a}$ & -696.9 & 796.9 & $1.5 \mathrm{~b}$ & 57.1 & 42.9 & $-1.01 b$ & -14.8 & 114.8 \\
\hline & $\mathrm{CK}$ & $89.3 \mathrm{c}$ & -- & 100.0 & $2.2 \mathrm{a}$ & -- & 100.0 & $-0.92 \mathrm{a}$ & -- & 100.0 \\
\hline \multirow[t]{3}{*}{$\begin{array}{c}\text { Fenyan } \\
\text { No.1 }\end{array}$} & $\mathrm{T} 1$ & $904.3 \mathrm{a}$ & -912.7 & 1012.7 & $1.6 \mathrm{~b}$ & 27.3 & 72.7 & $-1.03 c$ & -12.0 & 112.0 \\
\hline & $\mathrm{T} 2$ & $946.2 \mathrm{~b}$ & -959.6 & 1059.6 & $1.8 \mathrm{~b}$ & 18.2 & 81.8 & $-0.94 b$ & -2.2 & 102.2 \\
\hline & $\mathrm{CK}$ & 92.8 & -- & 100.0 & 3.1 & -- & 100.0 & -0.91 & -- & 100.0 \\
\hline \multirow[t]{2}{*}{ Average } & $\mathrm{T} 1$ & 739.6 & -696.9 & 796.9 & 1.4 & 54.3 & 45.7 & -1.00 & -10.3 & 110.3 \\
\hline & $\mathrm{T} 2$ & 873.8 & -841.6 & 941.6 & 1.6 & 48.9 & 51.1 & -0.97 & -7.0 & 107.0 \\
\hline
\end{tabular}


Table 3. Temperature effects on the activities and contents of six chemical substances in three tomato cultivars under weak light stress conditions from experiment two.

\begin{tabular}{|c|c|c|c|c|c|c|c|c|c|c|}
\hline Cultivar & Treatment & $\begin{array}{c}\text { SOD } \\
\text { (U/mg.protein) }\end{array}$ & $\begin{array}{l}\text { Relative } \\
\text { SOD } \\
\text { reduction } \\
\text { value (\%) }\end{array}$ & $\begin{array}{c}\text { Relative } \\
\text { SOD value } \\
\text { (CK as 100) }\end{array}$ & $\begin{array}{c}\text { POD } \\
(\mathrm{U} / \mathrm{mg} \cdot \text { protein } \cdot \mathrm{min})\end{array}$ & $\begin{array}{l}\text { Relative } \\
\text { POD } \\
\text { reduction } \\
\text { value (\%) }\end{array}$ & $\begin{array}{c}\text { Relative } \\
\text { POD value } \\
(\mathrm{CK} \text { as } 100)\end{array}$ & $\begin{array}{c}\text { MDA } \\
\text { (nmol/mg.FW) }\end{array}$ & $\begin{array}{l}\text { Relative } \\
\text { MDA } \\
\text { reduction } \\
\text { value (\%) }\end{array}$ & $\begin{array}{l}\text { Relative } \\
\text { MDA } \\
\text { value } \\
(\mathrm{CK} \text { as } \\
100)\end{array}$ \\
\hline \multirow{3}{*}{ SV0313TG } & CK & $16.7 \mathrm{a}$ & -- & 100.0 & $85.6 a$ & -- & 100.0 & $11.0 \mathrm{~b}$ & -- & 100.0 \\
\hline & $\mathrm{T} 1$ & $16.6 \mathrm{a}$ & 0.6 & 99.4 & $53.0 \mathrm{~b}$ & 38.1 & 61.9 & $11.0 \mathrm{~b}$ & 0.0 & 100.0 \\
\hline & $\mathrm{T} 2$ & $11.2 \mathrm{~b}$ & 32.9 & 67.1 & $20.2 \mathrm{c}$ & 76.4 & 23.6 & $23.2 \mathrm{a}$ & -110.9 & 210.9 \\
\hline \multirow{3}{*}{ Ousa } & $\mathrm{CK}$ & 21.1a & -- & 100.0 & $97.7 \mathrm{a}$ & -- & 100.0 & $11.8 \mathrm{~b}$ & -- & 100.0 \\
\hline & $\mathrm{T} 1$ & $15.8 \mathrm{a}$ & 25.1 & 74.9 & $59.6 \mathrm{~b}$ & 39.0 & 61.0 & $11.0 \mathrm{~b}$ & 6.8 & 93.2 \\
\hline & $\mathrm{T} 2$ & $16.3 a$ & 22.7 & 77.3 & $25.3 \mathrm{c}$ & 74.1 & 25.9 & $23.1 \mathrm{a}$ & -95.8 & 195.8 \\
\hline \multirow{3}{*}{ Fenyan No.1 } & CK & $24.4 \mathrm{a}$ & -- & 100.0 & $106.4 \mathrm{a}$ & -- & 100.0 & $13.2 \mathrm{a}$ & -- & 100.0 \\
\hline & $\mathrm{T} 1$ & $15.4 \mathrm{ab}$ & 36.9 & 63.1 & $49.7 \mathrm{~b}$ & 53.3 & 46.7 & $12.5 \mathrm{a}$ & 5.3 & 94.7 \\
\hline & $\mathrm{T} 2$ & $11.8 \mathrm{~b}$ & 51.6 & 48.4 & $20.6 \mathrm{~b}$ & 80.6 & 19.4 & $15.1 \mathrm{a}$ & -14.4 & 114.4 \\
\hline \multirow{3}{*}{ Average } & CK & 20.7 & -- & 100.0 & 96.6 & -- & 100.0 & 12.0 & -- & 100.0 \\
\hline & $\mathrm{T} 1$ & 15.9 & 23.2 & 76.8 & 54.1 & 44.0 & 56.0 & 11.5 & 4.2 & 95.8 \\
\hline & $\mathrm{T} 2$ & 13.1 & 36.8 & 63.2 & 22.0 & 77.2 & 22.8 & 20.5 & -70.6 & 170.6 \\
\hline \multirow[t]{2}{*}{ Cultivar } & Treatment & $\begin{array}{c}\text { Proline } \\
\mu \mathrm{g} /(\mathrm{g} \cdot \mathrm{FW})\end{array}$ & $\begin{array}{l}\text { Relative } \\
\text { Proline } \\
\text { reduction } \\
\text { value (\%) }\end{array}$ & $\begin{array}{c}\text { Relative } \\
\text { Proline } \\
\text { value } \\
\text { (CK as 100) }\end{array}$ & $\begin{array}{c}\text { Soluble sugar } \\
\mathrm{mg} /(\mathrm{g} \cdot \mathrm{FW})\end{array}$ & $\begin{array}{c}\text { Relative } \\
\text { Soluble } \\
\text { sugar } \\
\text { reduction } \\
\text { value (\%) }\end{array}$ & $\begin{array}{c}\text { Relative } \\
\text { Soluble sugar } \\
\text { value } \\
\text { (CK as 100) }\end{array}$ & $\begin{array}{l}\text { Osmotic } \\
\text { potential } \\
(\mathrm{Mpa})\end{array}$ & $\begin{array}{l}\text { Relative } \\
\text { Osmotic } \\
\text { potential } \\
\text { reduction } \\
\text { value (\%) }\end{array}$ & $\begin{array}{c}\text { Relative } \\
\text { Osmotic } \\
\text { potential } \\
\text { value } \\
(\mathrm{CK} \text { as } \\
100)\end{array}$ \\
\hline & CK & $47.6 \mathrm{~b}$ & -- & 100.0 & $1.5 \mathrm{c}$ & -- & 100.0 & $-1.11 \mathrm{c}$ & -- & 100.0 \\
\hline \multirow[t]{3}{*}{ SV0313TG } & $\mathrm{T} 1$ & $60.4 \mathrm{~b}$ & -26.9 & 126.9 & $2.5 \mathrm{~b}$ & -66.7 & 166.7 & $-1.03 b$ & 7.2 & 92.8 \\
\hline & $\mathrm{T} 2$ & $114.6 \mathrm{a}$ & -140.8 & 240.8 & $3.3 \mathrm{a}$ & -120.0 & 220.0 & $-0.98 \mathrm{a}$ & 11.7 & 88.3 \\
\hline & CK & $30.4 \mathrm{~b}$ & -- & 100.0 & $1.2 \mathrm{~b}$ & -- & 100.0 & $-1.13 c$ & -- & 100.0 \\
\hline \multirow[t]{3}{*}{ Ousa } & $\mathrm{T} 1$ & $34.6 \mathrm{~b}$ & -13.8 & 113.8 & $2.4 \mathrm{a}$ & -100.0 & 200.0 & $-1.04 \mathrm{a}$ & 8.0 & 92.0 \\
\hline & $\mathrm{T} 2$ & $122.9 \mathrm{a}$ & -304.3 & 404.3 & $2.4 \mathrm{a}$ & -100.0 & 200.0 & $-1.07 \mathrm{~b}$ & 5.3 & 94.7 \\
\hline & $\mathrm{CK}$ & $74.3 \mathrm{~b}$ & -- & 100.0 & $1.2 \mathrm{~b}$ & -- & 100.0 & $-1.08 b$ & -- & 100.0 \\
\hline \multirow[t]{3}{*}{ Fenyan No.1 } & $\mathrm{T} 1$ & $120.0 \mathrm{~b}$ & -61.5 & 161.5 & $2.3 \mathrm{a}$ & -91.7 & 191.7 & $-1.15 c$ & -6.5 & 106.5 \\
\hline & $\mathrm{T} 2$ & $145.9 a$ & -96.4 & 196.4 & $2.8 \mathrm{a}$ & -133.3 & 233.3 & $-0.98 \mathrm{a}$ & 9.3 & 90.7 \\
\hline & CK & 50.8 & -- & 100.0 & 1.3 & -- & 100.0 & -1.11 & -- & 100.0 \\
\hline \multirow[t]{2}{*}{ Average } & $\mathrm{T} 1$ & 71.7 & -41.2 & 141.2 & 2.4 & -84.6 & 184.6 & -1.07 & 3.0 & 97.0 \\
\hline & $\mathrm{T} 2$ & 127.8 & -151.7 & 251.7 & 2.8 & -117.9 & 217.9 & -1.01 & 8.7 & 91.3 \\
\hline
\end{tabular}


1000.0

900.0

800.0

700.0

600.0

500.0

400.0

300.0

200.0

100.0

$\mathbf{0 . 0}$

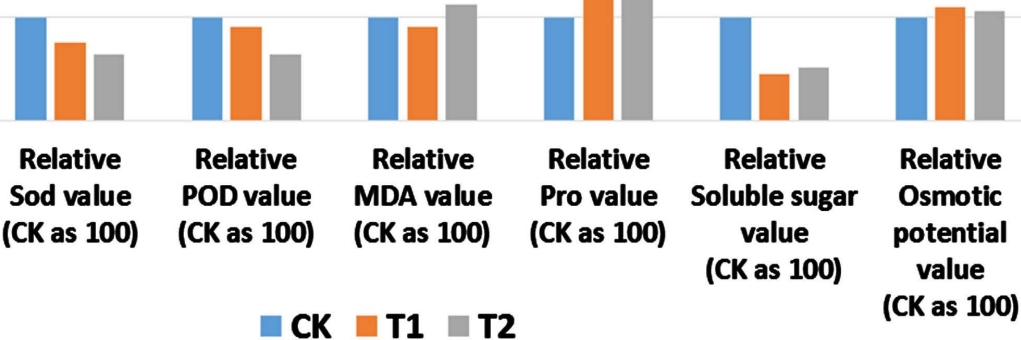

Figure 1. Effects of normal light under three different temperatures on the six chemical activities using relative values where the control (CK) as 100 .

respectively (Table 2, Figure 2); and SOD was decreased by $20.3 \%$ and $30.4 \%$, and POD by $10.9 \%$ and $30.9 \%$ under $\mathrm{T} 1$ and $\mathrm{T} 2$, respectively (Table 2 ).

Leaf MDA content was greatest for $\mathrm{T} 2$ and least for $\mathrm{T} 1$, and intermediate for CK. MDA activity decreased at T1, but increased at T2 condition, suggesting that low temperature decreased the MDA activity, but lowing the temperature to some extent, the MDA activity will increase, such as the relative average value decreased $8.9 \%$ at $\mathrm{T} 1$ and $-12.3 \%$ (i.e. increased by $12.3 \%$ ) at $\mathrm{T} 2$ (Table 2). When the CK was standardized at $100 \%$, the MDA value averaged was $91.1 \%$ and $112.3 \%$ under $\mathrm{T} 1$ and $\mathrm{T} 2$ conditions, respectively (Table 2, Figure 1). The two tomato cultivars, SV0313TG and Ousa had the same trends of T2 $>\mathrm{CK}>\mathrm{T} 1$ (Table 2, Figure 2 and Figure 3), but Fenyan No.1 showed a trend of T1 $<\mathrm{T} 2<$ $\mathrm{CK}$, indicating MDA decreased its activity under both low temperature stress in Fenyan No.1 (Table 2, Figure 4) but was different from other two did, indicating different tomato cultivars were affected by different extends for MDA activity 
800.0

600.0

400.0

200.0

$\mathbf{0 . 0}$

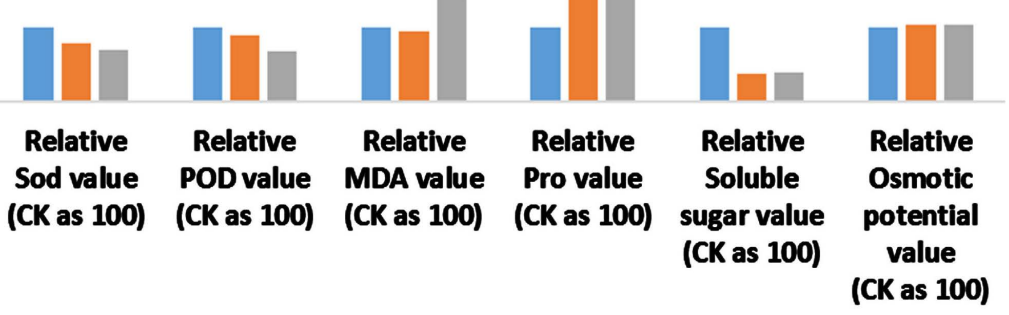

घV0313TG_CK $\backsim$ SV0313TG_T1 $\square$ SV0313TG_T2

Figure 2. Effects of normal light for tomato cultivar SV0313TG under three different temperatures on the six chemical activities using relative values where the control $(\mathrm{CK})$ as 100.

under low temperature stress.

Proline content was greatest with T2 and least for CK, and intermediate for T1. These results indicated that proline content increased as temperature decreased and under low temperature stress (Table 2). Averaged from the three cultivars, proline was increased by $696.9 \%$ and $841.6 \%$ under $\mathrm{T} 1$ and $\mathrm{T} 2$, respectively (Table 2). When the CK was standardized as $100 \%$, the average proline value was $796.9 \%$ and $941.6 \%$ under $\mathrm{T} 1$ and $\mathrm{T} 2$ conditions, respectively (Table 2, Figure 1), an 8 to 9 -fold increase. The three cultivars showed the same trends (Table 2, Figures 2-4). For example, when the CK was standardized at 100\%, the proline value in SV0313TG was $763.8 \%$ and $989.7 \%$ under T1 and T2 conditions, respectively (Table 2, Figure 2) and decreased by $-663.8 \%$ and $-889.7 \%$ (i.e. increased $663.8 \%$ and $889.7 \%$ ) under $\mathrm{T} 1$ and $\mathrm{T} 2$, respectively (Table 2).

Soluble sugars decreased as temperature decreased (Table 2). When averaged across all three cultivars, soluble sugar content decreased by $54.3 \%$ and $48.9 \%$ under T1 and T2 conditions, respectively (Table 2). When the CK was standardized as $100 \%$, the average soluble sugar content was $45.7 \%$ and $51.2 \%$ under $\mathrm{T} 1$ and T2 conditions, respectively (Table 2, Figure 1). The three cultivars showed 
800.0

700.0

600.0

500.0

400.0

300.0

200.0

100.0

0.0
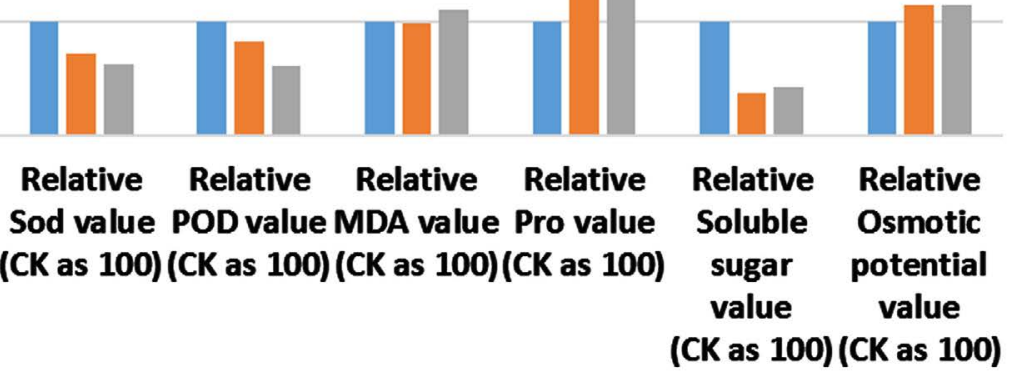

$\square$ Ousa_CK $\square$ Ousa_T1 $\square$ Ousa_T2

Figure 3. Effects of normal light for tomato cultivar Ousa under three different temperatures on the six chemical activities using relative values where the control (CK) as 100 .

the same trends (Table 2, Figures 2-4). For example, when the CK was standardized at $100 \%$, the soluble sugar value in SV0313TG was $37.1 \%$ and $40.0 \%$ under $\mathrm{T} 1$ and $\mathrm{T} 2$ conditions, respectively (Table 2, Figure 2) and decreased by $62.9 \%$ and $60.0 \%$ under $\mathrm{T} 1$ and $\mathrm{T} 2$, respectively (Table 2 ).

Lower temperatures resulted in increased osmotic potential in leaf tissues (Table 2), and these trends were similar across cultivars (Table 2, Figures 1-4). For example, when the CK was standardized as $100 \%$, the osmotic potential value in SV0313TG was 104.3\% under both T1 and T2 conditions (Table 2, Figure 1) and decreased by $-4.3 \%$ (i.e. increased $4.3 \%$ ) under both $\mathrm{T} 1$ and $\mathrm{T} 2$ (Table 2 ). When averaged across cultivars, osmotic potential increased by $10.3 \%$ and $7.0 \%$ under $\mathrm{T} 1$ and $\mathrm{T} 2$, respectively (Table 2 ).

\subsection{Temperature Effects on the Activity and Content of Six Chemical Substances in Tomato under Weak Light}

Concentrations of SOD and POD activity decreased as temperature decreased 
1000.0

800.0

600.0

400.0

200.0

0.0

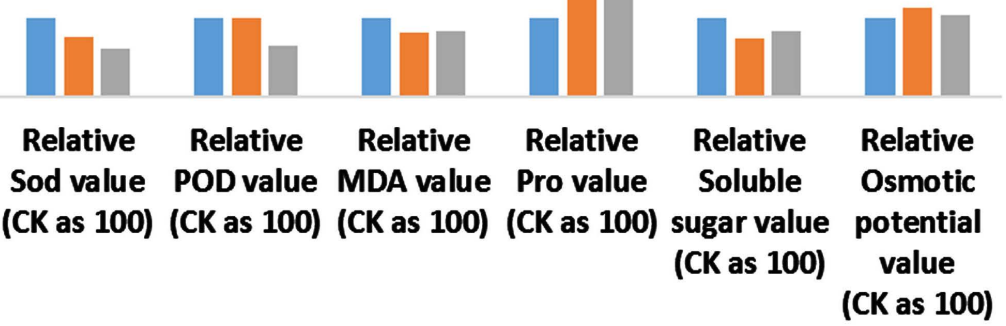

\section{Fenyan No.1_CK Fenyan No.1_T1 Fenyan No.1_T2}

Figure 4. Effects of normal light for tomato cultivar Fenyan No.1 under three different temperatures on the six chemical activities using relative values where the control $(\mathrm{CK})$ as 100 .

under low light intensity (Table 3). Cultivars showed similar trends overall, and SOD across cultivars decreased an average of $23.2 \%$ and $36.8 \%$, and POD $44.0 \%$ and $77.2 \%$ under $\mathrm{T} 1$ and $\mathrm{T} 2$, respectively (Table 3 ). When the CK was standardized as $100 \%$, the average SOD value was $76.8 \%$ and $63.2 \%$, and the POD was $56.0 \%$ and $22.8 \%$ under $\mathrm{T} 1$ and $\mathrm{T} 2$ conditions, respectively (Table 3, Figure 5). For example, when the CK was taken as 100, the SOD value in SV0313TG was 99.4 and 67.1, and the POD was 61.9 and 24.6 under T1 and T2 conditions, respectively (Table 3, Figure 2); and SOD was decreased by $0.6 \%$ and $32.9 \%$, and POD by $38.1 \%$ and $76.4 \%$ under $\mathrm{T} 1$ and $\mathrm{T} 2$, respectively (Table 3 ).

Leaf MDA content was greatest for T2 and lowest for T1, with CK as intermediate. These results indicated that MDA activity decreased slightly at T1, but increased at T2 condition, suggesting that low temperature decreased the MDA activity, but lowing the temperature to some extent, the MAD activity will increase, such as the relative average value decreased $4.2 \%$ at $\mathrm{T} 1$ and $-70.6 \%$ (i.e. increased by $70.6 \%$ ) at $\mathrm{T} 2$ (Table 3 ). When the $\mathrm{CK}$ was taken as 100 , the MDA 


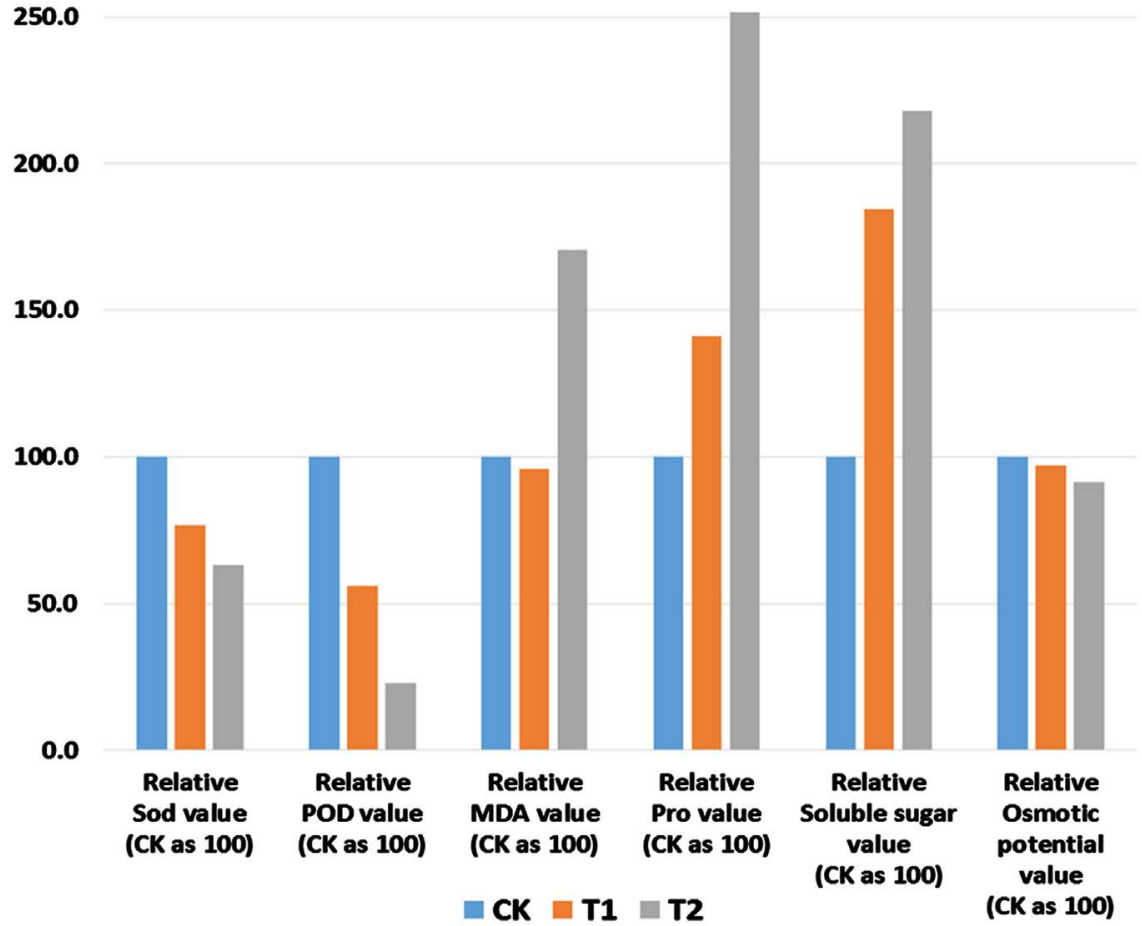

Figure 5. Effects of weak light under three different temperatures on the six chemical activities using relative values where the control (CK) as 100.

value averaged was 95.8 and 170.6 under T1 and T2 conditions, respectively (Table 3, Figure 1). The two tomato cultivars had the same trends $\mathrm{T} 2>\mathrm{CK}>\mathrm{T} 1$ as the averaged (Table 3, Figures 6-8).

For the proline content, $\mathrm{T} 2>\mathrm{T} 1>\mathrm{CK}$, indicating under low temperature stress, proline content increased and the lower temperature was, the more proline content increased (Table 3). Averaged from the three cultivars, proline was increased by $41.2 \%$ and $151.7 \%$ under $\mathrm{T} 1$ and $\mathrm{T} 2$, respectively (Table 3 ). When the CK was taken as 100, the average proline value was 141.2 and 251.7 under T1 and T2 conditions, respectively (Table 3, Figure 5). The three cultivars showed the same trends as the averaged (Table 3, Figures 6-8). For example, when the CK was taken as 100, the proline value in SV0313TG was 126.9 and 240.8 under $\mathrm{T} 1$ and $\mathrm{T} 2$ conditions, respectively (Table 3, Figure 6 ) and decreased by $-26.9 \%$ and $-140.8 \%$ (i.e. increased $26.9 \%$ and $140.8 \%$ ) under $\mathrm{T} 1$ and $\mathrm{T} 2$, respectively (Table 3).

For the soluble sugar content, T2 $>\mathrm{T} 1>\mathrm{CK}$ under weak light conditions, indicating under low temperature stress, soluble sugar content increased (Table 3). Averaged from the three cultivars, the soluble sugar content was increased by $84.6 \%$ and $117.9 \%$ under $\mathrm{T} 1$ and $\mathrm{T} 2$, respectively (Table 3 ). When the CK was 
weak light

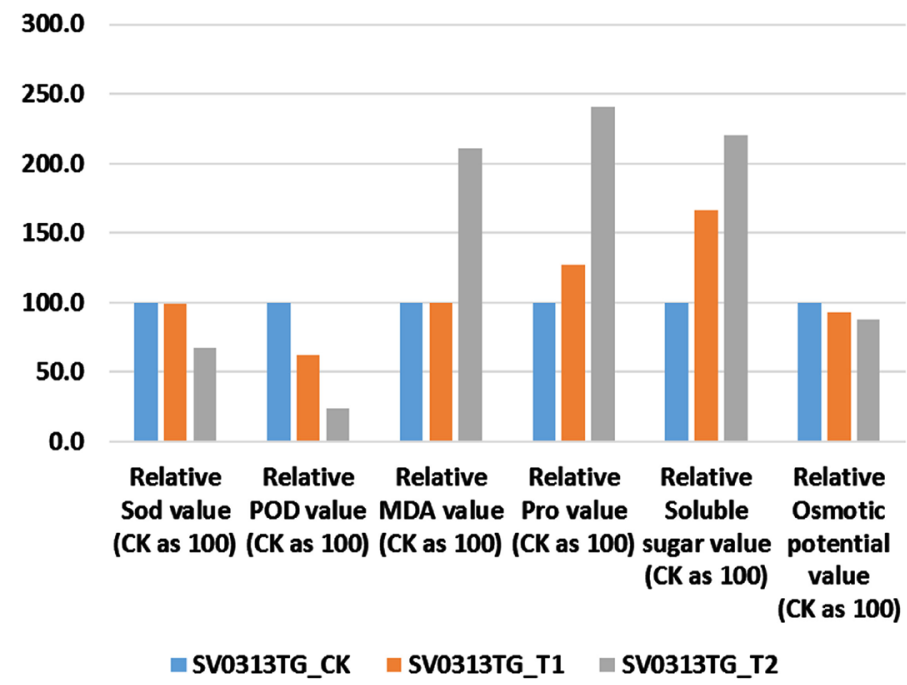

Figure 6. Effects of weak light for tomato cultivar SV0313TG under three different temperatures on the six chemical activities using relative values where the control (CK) as 100 .

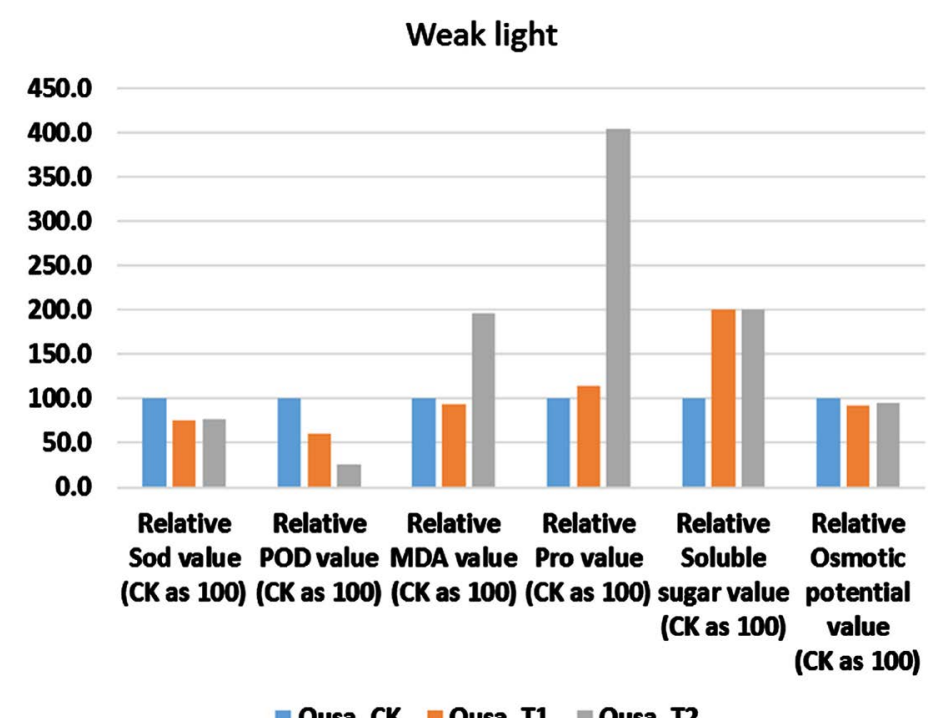

Figure 7. Effects of weak light for tomato cultivar Ousa under three different temperatures on the six chemical activities using relative values where the control (CK) as 100.

taken as 100 , the average soluble sugar value was 184.6 and 217.9 under T1 and T2 conditions, respectively (Table 3 , Figure 5 ). The three cultivars showed the same trends as the averaged (Table 3, Figures 6-8). For example, when the CK was taken as 100, the soluble sugar value in SV0313TG was 166.7 and 220.0 under $\mathrm{T} 1$ and $\mathrm{T} 2$ conditions, respectively (Table 3, Figure 6) and increased by $66.7 \%$ and $120.0 \%$ under $\mathrm{T} 1$ and $\mathrm{T} 2$, respectively (Table 3 ).

For the osmotic potential content, T1 (T2) < CK, indicating under low temperature stress, osmotic potential content decreased (Table 3). The three cul- 


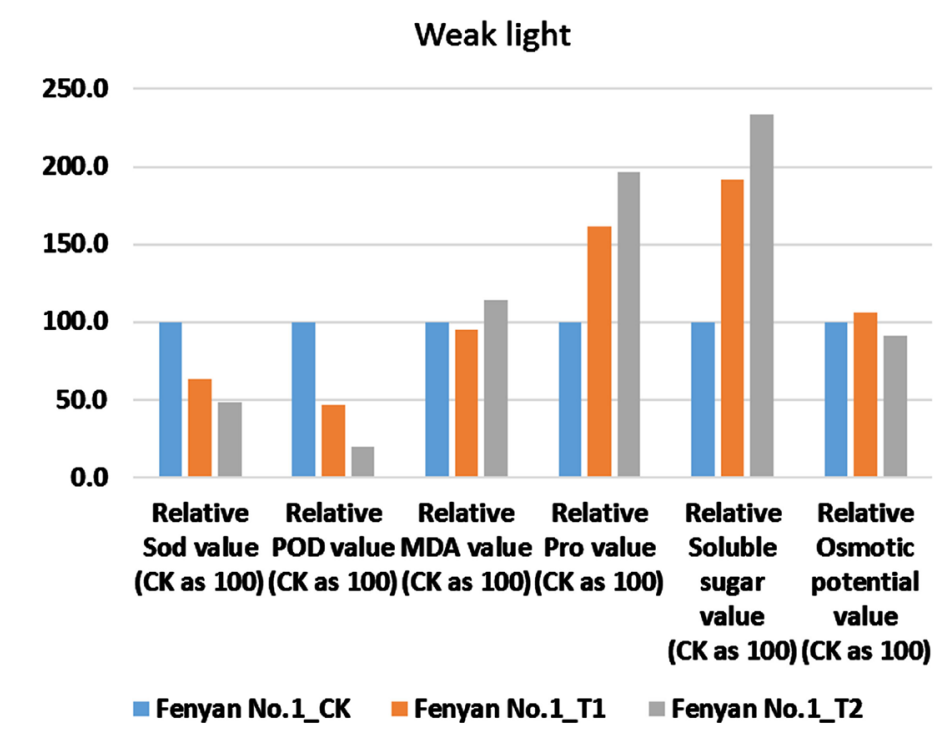

Figure 8. Effects of weak light for tomato cultivar Fenyan No.1 under three different temperatures on the six chemical activities using relative values where the control (CK) as 100 .

tivars individually or averaged showed similar trends (Table 3, Figures 5-8). For example, when the CK was taken as 100, the osmotic potential value in SV0313TG was 92.8 and 88.3 under both T1 and T2 conditions (Table 3, Figure 5) and decreased by $7.2 \%$ and $11.7 \%$ under both $\mathrm{T} 1$ and $\mathrm{T} 2$ (Table 3 ). Averaged from the three cultivars, the osmotic potential was decreased by $3.0 \%$ and $8.7 \%$ under $\mathrm{T} 1$ and $\mathrm{T} 2$, respectively (Table 3 ). When the CK was taken as 100 , the average osmotic potential value was 97.0 and 91.3 (Table 3 and Figure 6).

\section{Discussion}

Tomato growth and development require stronger light and higher temperatures. The light saturation point is generally between 30,000 and 40,000 lux, and the suitable temperature is around $25^{\circ} \mathrm{C}$ [3] [8]. Two experiments were conducted in this study: the Expt. 1 under normal light and Expt. 2 under weak light with three temperature sets. Through the two experiments, the physiological response mechanism of tomato seedlings treated with different low temperatures and weak light was studied to further explore the cold tolerance mechanism at tomato seedlings.

SOD is one of the most important enzymes for scavenging reactive oxygen free radical in plant cells. Its main function is to scavenge $\mathrm{O}_{2}^{-}$and produce $\mathrm{H}_{2} \mathrm{O}_{2}$. Although it is not reactive oxygen radical, $\mathrm{H}_{2} \mathrm{O}_{2}$ can interact with $\mathrm{O}_{2}^{-}$. More oxygen-free radicals pose greater harm to cells; while POD has the effect of breaking down $\mathrm{H}_{2} \mathrm{O}_{2}$, thereby reducing the damage to cells [19]. In this study, SOD and POD activities decreased in both experiments under normal and weak light conditions, as the temperature decreased, and the lower temperature was, the more activities of both enzymes in three tomato cultivars either individual or averaged decreased (Table $2 \&$ Table 3, Figures 1-8). Under low temperature 
treatment, due to the excessive production of active oxygen free radicals in the leaves, some of the reactive oxygen free radicals in the leaves exceeded the threshold. Some active oxygen free radicals with strong oxidizing ability had direct damage to SOD, which caused a decrease of SOD activity in the leaves [20]. Under the condition of weak light stress, SOD and POD activities of the two antioxidant enzymes decreased and decreased more under lower temperatures. The results were consistent with previous studies [21] that the worse environmental conditions of stress in plants, the greater the reduction in SOD and POD values.

Under the adverse conditions, excessive free radicals in the plant and both bonds of unsaturated fatty acids in the structure of the active oxygen oxide membrane cause the chain to break, decompose and destroy. The reaction occurs on the membrane structure, which is membrane lipid peroxidation, and the final product is MDA [22] and its content can reflect the degree of membrane damage to some extent [23] [24]. The MDA itself is cytotoxic, and it can bind to proteins and enzymes on membrane structure, cross-link to make it inactive, further destroy cell membrane structure [22] [25]. The low temperature stress has certain damage to plant cell plasma membrane [26] and it will inevitably cause changes in MDA content. The relative content of MDA was consistent in both experiments (Figure 4 and Figure 8). Under the normal illumination and low temperature treatments, the $\mathrm{T} 1$ value decreased, and the relative decrease was $8.9 \%$. The $\mathrm{T} 2$ value increased, and the relative increase was $12.3 \%$. Under both low light and low temperature treatments, the MDA value decreased under $\mathrm{T} 1$ condition with a relative decrease of $4.2 \%$; but increased $70.6 \%$ under the lower temperature of T2 condition. Previous studies suggested that the MDA content increased as the treatment temperature decreased, indicating that the degree of peroxidation of plant membranes increased with low temperature stress and the content of MDA and the cold tolerance of plants was negatively correlated to some extent [27]. Other studies showed that when the temperature was lowered, MDA content decreased first and then increased. The early decline of MDA content was in the initial stage of low temperature treatment. Generally, low temperature caused plants to undergo self-protection. As the low temperature stress enforced with lower temperature conditions, the MDA decreased greatly. In recent years, studies have raised questions about whether cell membrane phase transitions are altered under low temperature stress or at low temperature threshold values to be further tested [9] [28] [29]. Under the T1 condition when the temperature was not particularly low, the MDA content decreased a little. The plant may have its own protective mechanism, but under the T2 condition, the MDA content increased. It needs to be further validated whether the diurnal temperature $\left(15^{\circ} \mathrm{C} / 5^{\circ} \mathrm{C}\right)$ is a temporary temperature causing the cell membrane phase transition.

In the adversity, the content of soluble sugar and proline contents in the osmotic adjustment substance should be continuously accumulated to reduce the water potential of the cells and reduce the water loss caused by low temperature stress, and to protect crops from harm under adverse conditions [29] [30]. In the 
Expt. 1, the relative content of proline increased gradually with the decrease of temperature; the relative content of soluble sugar decreased compared to the $\mathrm{CK}$; and the relative value of osmotic potential increased (Table 2; Figures 1-4). In the case of weak light, as the temperature decreased; the relative contents of proline and soluble sugar contents increased gradually, and the relative value of the osmotic potential gradually decreased (Table 3; Figures $5-8$ ). The relative content of proline increased in both experiments but increased much greater in Expt. 1 (Table 2 and Table 3; Figures 1-8). The relative content of soluble sugar decreased in Expt. 1, but increased in Expt. 2 (Table 2 and Table 3; Figures 1-8). The relative content of osmotic potential increased in Expt. 1, but decreased in Expt. 2 (Table 2 and Table 3; Figures 1-8). When subjected to low temperature stress, the relative content of proline increased significantly; the relative content of soluble sugar decreased slightly; and the relative content of osmotic potential increased. When exposed to double stresses under low temperature and low light, the relative contents of proline and soluble sugar increased significantly. However, the increase of proline was less than that of only low temperature stress, and the relative content of osmotic potential decreases. At low temperature, the plant adjusted the cell osmotic potential by adjusting its own osmotic adjustment substance, and the proline-based soluble regulatory substance content increased, and the relative content of the osmotic potential increased, thereby protecting cell activity and improving its resistance. Under low light stress, the contents of proline and soluble sugar increased, so as to improve their resistance to stress, but the double stresses caused the plant's osmotic potential to decrease.

Accumulation of sugar content in plant leaves under low temperature stress is a common phenomenon. The accumulation of sugar can increase the water retention capacity and increase the cell osmotic concentration to make it more resistant to cold [31]. There was a positive correlation between the content of soluble sugars and the cold resistance of plants. Other reports showed under low temperature stress, the soluble sugar in the plants would increase and the cold tolerant cultivars had a stronger resistance to cold than those with weak cold resistance in grape, cucumber and wheat [32] [33] [34]. According to Levitt [35], in the cold damage, especially in critical low temperature or lethal temperature condition, as long as the tissue of the plant was not killed, the sugar in the plant cells can continue to accumulate. The increase of soluble sugar content can increase the osmotic pressure of the cell liquid so that the cell liquid does not leak out, causing the cells not to lose water in order not to reduce the number of ice crystals. In this study, the soluble sugar content was significantly increased under both low temperature and low light conditions, which was consistent with the results reported by [35]. Soluble sugar showed some hysteresis compared with other osmotic adjustment substances, but the physicochemical properties of sugar determined its osmotic adjustment with higher ability than any other substance [36]. However, there were other different opinions that the soluble sugar content of plants had no correlation with its cold tolerance [33]. In this study, 
under normal light in Expt. 1, during the low temperature, why the relative content of soluble sugars decreased needs to be further study.

Under stress, the plant should continue to absorb water from the medium with low water potential to maintain the water balance in the body, and the swell pressure basically to ensure the normal operation of the physiological and biochemical processes in the body in order to reduce the osmotic potential through osmotic adjustment [37]. In this study, under low temperature stress, the plant body only regulated the osmotic potential by the increase of proline content, and the increased osmotic potential can make a large amount of soluble regulatory substances to maintain swell pressure. Under double stresses of the low temperature and weak light conditions, both soluble sugar and proline accumulation simultaneously, and the relatively reduced osmotic potential can avoid the excessive growth of osmotic adjustment substances and increase the swell pressure in the cells to protect plants to survive.

\section{Conclusion}

In summary, the activity of superoxide dismutase (SOD) and peroxidases (POD) in tomato seedlings decreased under low temperature stress in both experiments under normal and weak light conditions and the lower temperature, the more decreased in either the three tomato cultivars individually or averaged. The POD activity decreased more under double stress conditions with a low temperature and weak light. Malondialdehyde (MDA) increased only at the lower temperature T2 conditions under both experiments. Proline contents increased at both low temperatures under both experiments and the lower temperature was, the more its contents increased. The soluble sugar content decreased at both low temperatures under Experiment 1 under normal light condition but increased under weak light stress condition. The osmotic potential increased at low temperature stress under normal light but decreased under weak light stress conditions. This study will provide useful information to select tolerant tomato cultivars/lines under low temperature and weak light stress conditions.

\section{Conflicts of Interest}

The authors declare no conflicts of interest regarding the publication of this paper.

\section{References}

[1] Nie, C. and Song, J. (2008) Current Status of Main Vegetable Cultivation in Hebei Province. Chinese Vegetables, 4, 10-11.

[2] Zhu, J., Yang, Z. and Li, Y. (2012) Effects of High Temperature Stress on Photosynthetic Characteristics and Antioxidant Enzyme Activities of Tomato and Cucumber in Greenhouse. Northern Horticulture, No. 1, 63-68.

[3] Dong, L., Yang, Y. and Shi, L. (2016) Effects of Low Temperature and Low Light on Different Varieties of Tomato. Journal of North China Agriculture, 31, 183-187.

[4] Fu, R., Gao, S. and Xu, D. (2008) Effects of Low Temperature-Related Physiological 
Indicators on Melons. Fujian Resurgence Technology, 3, 1-2.

[5] Ma, Y. and Dai, X. (2000) Effects of Physiological Indexes Related to Cold Tolerance of Pepper under Low Temperature Stress. Journal of Hunan Agricultural University: Natural Science Edition, 26, 263-264.

[6] Zhou, Y., Yu, J. and Qian, Q. (2003) Effects of Low Temperature and Low Light on Growth and Antioxidant Enzyme Activities of Cucumber Seedlings. Acta Biologa Sinica, 14, 921-924.

[7] Zheng, Y., Yang, T. and Wei, A. (2008) Effects of Low Temperature Stress on Several Cold Physiological Indexes of Apricots. Journal of Northwest A\&F University (Natural Science Edition), 36, 460-465.

[8] Meng, L. and Jiang, Y. (2007) Differences in Physiological Adaptation of Tomato Seedlings to Low Temperature and Low Light Stress. Southwest Agricultural University, 20, 40-44.

[9] Wu, X., Yan, X. and Wu, L. (2009) Screening of Light and Temperature Environment in Greenhouses in Spring and the Low Temperature and Low Light Index of Tomato in Hefei Area. Chinese Agricultural Science Bulletin, 25, 162-166.

[10] Chen, H., He, X. and Li, X. (2010) Effects of Low Temperature Stress on Cold Resistance of Young Larvae of Longan. Guangxi Agricultural Sciences, 1, 13-16.

[11] Crowe, J.H., Hoekstra, F.A. and Crowe, L.M. (1992) Anhydrobiosis. Annual Review of Physiology, 54, 579-599. https://doi.org/10.1146/annurev.ph.54.030192.003051

[12] Ding, C., Yang, Q. and Li, F. (2005) Effects of Low Temperature Stress on Free Proline Content in Leaves of $C$. chinensis and P. glabra L. Journal of Tropical Crops, 26, 52-56.

[13] Huang, W., Ren, H. and Zhang, F. (2002) Effects of Low Temperature and Low Light on Growth and Photosynthesis of Tomato Seedlings. Chinese Vegetables, 1, 15-17.

[14] Zhao, K. (1993) Plant Salt Resistance Physiology. China Science and Technology Press, Beijing.

[15] Wu, W. (2003) Plant Physiolog. Beijing Science Press, Beijing.

[16] Zhang, Y. (2011) Plant Physiology Experiment Technology Course. Science Press, Beijing.

[17] Zhang, Z. and Yan, W. (2003) Experimental Guidance of Plant Physiology. Higher Education Press, Beijing.

[18] Li, H. (2000) Principles and Techniques of Plant Physiological and Biochemical Tests. Higher Education Press, Beijing.

[19] Scandalios, J.G. (1993) Oxygen Stress and Superoxide Dismutases. Plant Physiology, 101, 7. https://doi.org/10.1104/pp.101.1.7

[20] Hu, W. and Li, X. (2003) Effects of Low Temperature and Weak Light on Antioxidant Enzyme System in Tomato Leaves and Roots. Journal of Jinggangshan Normal University, 24, 19-22.

[21] Feng, J., Zhang, Y. and Yang, T. (2002) Effects of Low Temperature Stress on SOD Activity, MDA and Proline Content in Camptotheca acuminata Seedlings. Forestry Science Research, 15, 197-202.

[22] Sun, C. and Liu, J. (1998) Effects of Low Temperature Stress on $\mathrm{O}^{2-}$, MDA, Membrane Permeability, Leaf Water Potential and Protective Enzymes in Leaves of Populus euphratica. Journal of Inner Mongolia Forestry University, 20, 32-36.

[23] Jian, L. (1992) New Developments in the Mechanism of Cold Resistance of Plants. 
Bulletin, 9, 17-22.

[24] Zhang, Y. (2008) Changes in Physiological Characteristics of Tomato under Low Temperature and Low Light. Northern Horticulture, 3, 38-39.

[25] Liu, H., Zeng, Y. and Wang, Y. (1985) Effect of Low Temperature on Superoxide Dismutase in Various Organelles of Cucumber Seedlings with Different Cold Resistance. Chinese Journal of Plant Physiology, 11, 48-57.

[26] Wang, Z. and Tang, M. (2016) Effect of Low Temperature Exercise on Cold Resistance of White Palm Tissue Culture Seedlings. Journal of Southern Agricultural Sciences, 47, 1720-1724.

[27] Wang, Q., Luo, H. and Zhao, L. (2018) Evaluation of Cold Resistance of 24 Pomegranate Varieties. Shandong Agricultural Sciences, 50, 50-54, 59.

[28] Li, J. (2012) Effects of Low Temperature and Low Light on Eggplant Growth and Physiological and Biochemical Functions in Sunlight Greenhouse. Jilin Agricultural University, Changchun.

[29] Zhou, Y. and Nie, S. (2001) Study on the Physiological and Ecological Resistance of Cold Spring in Cuinan. Northeast Forestry University, Harbin.

[30] Pearce, R.S. (1999) Molecular Analysis of Acclimation to Cold. Plant Growth Regulation, 29, 47-76. https://doi.org/10.1023/A:1006291330661

[31] He, H., Tian, L. and Xue, L. (2007) Progress in Physiological and Biochemical Studies of Plant Cold Resistance. Tianjin Agricultural Sciences, 13, 10-13.

[32] Wang, S., Wang, J. and Li, Y. (1996) Study on the Relationship between Soluble Protein, Soluble Sugar Content and Cold Resistance of Grape. Northern Horticulture, 107, 13-14.

[33] Liu, Z. and Zhang, S. (1994) Plant Physiology. China Agriculture Press, Beijing.

[34] Sun, A. and Sun, Q. (1985) Overview of Research on Wheat Cold Resistance Physiology. Foreign Agronomy and Wheat Crops, 6, 32-35.

[35] Levitt, J. (1980) Responses of Plants to Environmental Stress, Volume 1: Chilling, Freezing, and High Temperature Stresses. Academic Press, Cambridge. https://doi.org/10.1016/B978-0-12-445501-6.50016-6

[36] Yu, T., Qin, L. and Wang, Y. (1996) The Studies of Accumulation of Solubility Sugars and Component Changes in Chinese Chestnut under Osmotic Stress. Journal of Beijing Agriculture College, 11, 43-47.

[37] Liang, Z., Li, M. and Wang, J. (1998) Sea Buckthorn Anti-Drought Physiological Mechanism. Hippophae, 11, 8-13. 